\title{
Incidental Findings Suggestive of COVID-19 in Asymptomatic Patients Undergoing Nuclear Medicine Procedures in a High-Prevalence Region
}

\author{
Domenico Albano ${ }^{1,2}$, Francesco Bertagna ${ }^{1,2}$, Mattia Bertoli ${ }^{2}$, Giovanni Bosio ${ }^{2}$, Silvia Lucchini ${ }^{2}$, Federica Motta ${ }^{2}$, \\ Maria Beatrice Panarotto ${ }^{2}$, Alessia Peli ${ }^{2}$, Luca Camoni ${ }^{2}$, Frank M. Bengel ${ }^{3}$, and Raffaele Giubbini ${ }^{1,2}$ \\ ${ }^{I}$ Nuclear Medicine, University of Brescia, Brescia, Italy; ${ }^{2}$ Nuclear Medicine, Spedali Civili Brescia, Brescia, Italy; and ${ }^{3}$ Department \\ of Nuclear Medicine, Hannover Medical School, Hannover, Germany
}

\begin{abstract}
Infection with the novel severe acute respiratory syndrome coronavirus 2 (SARS-CoV-2) may remain asymptomatic, leading to underrecognition of the related disease, coronavirus disease, 2019 (COVID-19), and to incidental findings in nuclear imaging procedures performed for standard clinical indications. Here, we report about our local experience in a region with high COVID-19 prevalence and dynamically increasing infection rates. Methods: Within the 8d period of March 16-24, 2020, hybrid imaging studies of asymptomatic patients who underwent ${ }^{18} \mathrm{~F}-\mathrm{FDG}$ PET/CT or ${ }^{131}$ I SPECT/CT for standard oncologic indications at our institution in Brescia, Italy, were analyzed for findings suggestive of COVID-19. The presence, radiologic features, and metabolic activity of interstitial pneumonia were identified, correlated with the subsequent short-term clinical course, and described in a case series. Results: Six of 65 patients (9\%) who underwent PET/CT for various malignancies showed unexpected signs of interstitial pneumonia on CT and elevated regional ${ }^{18} \mathrm{~F}-\mathrm{FDG}$ avidity. Additionally, 1 of 12 patients who received radioiodine for differentiated thyroid carcinoma also showed interstitial pneumonia on SPECT/CT. Five of 7 patients had subsequent proof of COVID-19 by reverse-transcriptase polymerase chain reaction. The remaining 2 patients were not tested immediately but underwent quarantine and careful monitoring. Conclusion: Incidental findings suggestive of COVID-19 may not be infrequent in hybrid imaging of asymptomatic patients in regions with an expansive spread of SARS-CoV-2. Nuclear medicine services should prepare accordingly.
\end{abstract}

Key Words: COVID-19; PET/CT; pneumonia; nuclear medicine

J Nucl Med 2020; 61:632-636

DOI: $10.2967 /$ jnumed.120.246256

A aggressive disease called coronavirus disease, 2019 (COVID-19), caused by severe acute respiratory syndrome coronavirus 2 (SARS-CoV-2), a novel coronavirus of zoonotic origin,

\footnotetext{
Received Mar. 31, 2020; revision accepted Mar. 31, 2020.

For correspondence or reprints contact: Domenico Albano, Nuclear Medicine, Spedali Civili di Brescia, Piazzale Spedali Civili 1, 25123 Brescia, Italy.

E-mail: doalba87@libero.it

Published online Apr. 1, 2020.

Immediate Open Access: Creative Commons Attribution 4.0 International License (CC BY) allows users to share and adapt with attribution, excluding materials credited to previous publications. License: https://creativecommons. org/licenses/by/4.0/. Details: http://jnm.snmjournals.org/site/misc/permission. xhtml.

COPYRIGHT @ 2020 by the Society of Nuclear Medicine and Molecular Imaging.
}

occurred initially in December 2019 in Wuhan, China, and then spread globally, becoming a pandemic. After the initial peak in China, the next countries to be severely affected were in Europe, especially Northern Italy, with a dramatic increase in cases of pneumonia and respiratory distress syndrome $(1,2)$. Although a severe course of COVID-19 is thought to occur in only a subfraction of infected individuals, many may present with nonspecific symptoms such as fever, cough, and dyspnea, and others may be asymptomatic initially or may remain asymptomatic entirely. The latter subjects are thought to be significant contributors to propagation of infection in the population.

Several reports are present in the literature (3-5) about the appearance of COVID-19 pneumonia on radiologic scans, such as chest radiography and CT. CT findings are typically characterized by ground-glass opacities (GGOs) or bilateral pulmonary consolidations in multiple lobular and subsegmental areas $(2,6)$. Currently, reverse-transcriptase polymerase chain reaction (RTPCR) from pharyngeal swabs is considered to be the gold standard for the diagnosis of COVID-19 infection, but a high false-negative rate has been reported and can cause the diagnosis to be missed or delayed, increasing the risk that the epidemic will spread (7). Explanations for the false-negative reports include a lack of shared and standardized operating procedures, a lack of validation across different laboratories and hospitals, differences in the stage of infection at the time of examination, and differences in viral load and in the potential mutation rate of the virus. For this reason, some authors suggest that a combination of clinical, imaging, and laboratory results should be used to establish the final diagnosis $(8,9)$.

Although nuclear medicine procedures are unlikely to play a role in the primary diagnosis of COVID-19, the disease may be detected incidentally in asymptomatic but infected patients undergoing scans for other indications, with potentially relevant implications for further management. Patterns of COVID-19 pneumonia on ${ }^{18} \mathrm{~F}$-FDG PET/CT have recently been anecdotally described for symptomatic patients (10-12). The aim of the present case series is to describe our local experience in Northern Italy, triggered by an increase in incidental detection of pneumonia suspected of stemming from COVID-19 in our routine nuclear medicine services.

\section{MATERIALS AND METHODS}

Between March 16 and March 24, 2020, $65{ }^{18}$ F-FDG PET/CT studies were performed for routine oncologic indications in our Department of Nuclear Medicine in Brescia, Italy. Because of the concurrent 


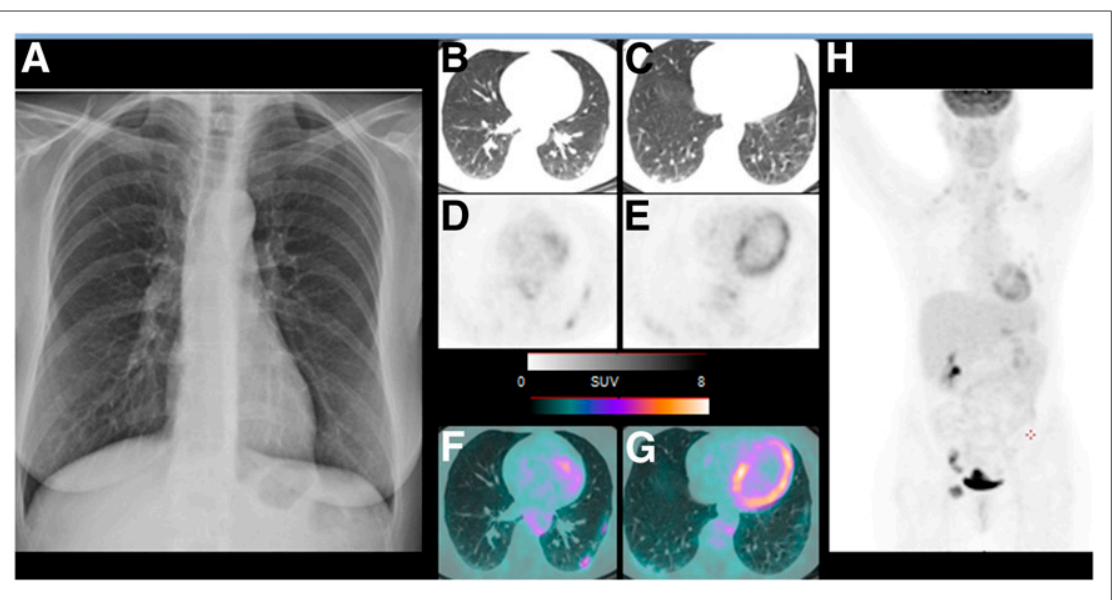

FIGURE 1. (A) Chest radiographs without pathologic findings. (B-G) Subsequent axial $C T$ (B and $\mathrm{C})$, PET ( $\mathrm{D}$ and $\mathrm{E}$ ), and PET/CT ( $\mathrm{F}$ and $\mathrm{G}$ ) images showing pleural consolidative areas that are ${ }^{18} \mathrm{~F}-$ FDG-positive $\left(\mathrm{SUV}_{\max }, 3.6\right)$. $(\mathrm{H})$ Maximum-intensity projection showing hypermetabolic inguinal lesions.

high prevalence and increasing dynamics of coronavirus infections in the region, all patients were carefully screened by triage before general access to the hospital, and again before entering the nuclear medicine service unit, and were asymptomatic. Accordingly, no suspicion of viral infection was present at the time of PET/CT in any patient. According to standard operating procedures, ${ }^{18} \mathrm{~F}-\mathrm{FDG}$ PET/CT was performed after a fast of more than $6 \mathrm{~h}$, with blood glucose levels less than $150 \mathrm{mg} / \mathrm{dL}$. A 4.5 MBq/kg activity was administered intravenously, and imaging was performed $60 \mathrm{~min}$ after injection from the skull base to the mid thigh (4 min per bed position, in steps of $15 \mathrm{~cm}$ ) using a Discovery ST or 690 scanner (GE Healthcare). For both scanners, a standard non-contrastenhanced free-breathing helical CT scan was obtained for morphologic correlation and attenuation correction. The Discovery ST acquisition parameters were $120 \mathrm{kV}$, a fixed tube current of $80 \mathrm{~mA}, 8$ slices $3.75 \mathrm{~mm}$ thick at $3.27-\mathrm{mm}$ intervals, a pitch of $1: 5$, and a tube rotation of $0.8 \mathrm{~s}$. The Discovery 690 acquisition parameters were $120 \mathrm{kV}$, a fixed tube current of $80 \mathrm{~mA}, 64$ slices $3.75 \mathrm{~mm}$ thick at 3.27 -mm intervals, a pitch of 0.984:1, and a tube rotation of $0.5 \mathrm{~s}$. The lung CT images were reconstructed using a $512 \times 512$ matrix and an iterative method, a slice thickness of $1.25 \mathrm{~mm}$ (Discovery 690) or $2.5 \mathrm{~mm}$ (Discovery STE), an interval of $1.25 \mathrm{~mm}$, and a lung filter with the window set at a width of 1,600 Hounsfield units and the level set at -480 Hounsfield units.

During the same period, 12 patients were admitted for radioiodine ablation of the thyroid remnant after thyroidectomy for differentiated thyroid carcinoma. All patients underwent whole-body ${ }^{131}$ I scintigraphy followed by SPECT/CT 3-4 d after radioiodine administration, using a dual-head camera (Infinia Hawkeye II; GE Healthcare) equipped with 2.54-cm (1-in) crystals and a high-energy collimator. The CT scan was acquired during free breathing, and the parameters were $140 \mathrm{kV}$, $2.5 \mathrm{~mA}$, a 30-s rotation time in axial mode, 4 slices $5 \mathrm{~mm}$ thick, and a $256 \times 256$ matrix.

\section{RESULTS}

On PET/CT images, 6 of 65 patients (9\%) showed CT signs of interstitial pneumonia suggestive of COVID-19. In all cases, the areas of pneumonia were ${ }^{18} \mathrm{~F}-\mathrm{FDG}-$ avid. This frequency of incidental pneumonia was significantly higher than that for the same period $1 \mathrm{y}$ earlier, before the onset of the COVID-19 pandemia (only 2/80 PET/CT studies performed during these $8 \mathrm{~d}$ in March 2019 showed incidental pneumonia).

\section{Case 1}

An asymptomatic 56-y-old woman underwent ${ }^{18} \mathrm{~F}-\mathrm{FDG}$ PET/CT for primary staging of anal cancer after recent primary surgical therapy (February 2020). Chest radiography before surgery was negative for any lung alterations (Fig. 1A). PET/CT identified ${ }^{18} \mathrm{~F}$-FDG-avid inguinal nodal metastases but also showed ${ }^{18} \mathrm{~F}-\mathrm{FDG}$-positive GGOs and consolidation in both lungs, most pronounced in the inferior lobes (Figs. 1B-1E). RT-PCR testing for SARS-CoV-2 was initiated and confirmed the diagnosis of COVID-19. Subsequent oncologic therapy was postponed, and the patient started COVID-19 therapy with hydroxychloroquine plus ritonavir-lopinavir.

\section{Case 2}

An asymptomatic 77-y-old man underwent ${ }^{18} \mathrm{~F}-\mathrm{FDG}$ PET/CT for staging of laryngeal cancer before therapy. PET/CT showed increased ${ }^{18} \mathrm{~F}$ FDG uptake at the right epiglottis and local cervical nodes (Fig. $2 \mathrm{~A})$. Additionally, there was bilateral faint ${ }^{18} \mathrm{~F}$-FDG uptake in GGOs not suggestive of aspiration (Figs. 2B-2G). RT-PCR testing was initiated immediately and confirmed the diagnosis of COVID19. Subsequent surgical therapy was postponed, and the patient began receiving therapy with hydroxychloroquine plus ritonavirlopinavir.

\section{Case 3}

An asymptomatic 55-y-old woman with a history of invasive ductal breast carcinoma previously treated by surgery, chemotherapy, and radiotherapy underwent ${ }^{18} \mathrm{~F}$-FDG PET/CT for restaging. A high-resolution chest $\mathrm{CT}$ scan 2 wk earlier showed a suggestive retrosternal lymph node but no lung disease (Figs. 3A and 3B). PET confirmed retrosternal ${ }^{18} \mathrm{~F}-\mathrm{FDG}$-avid nodal relapse and showed novel GGOs in the posterior segments of the inferior lung lobes (Figs. 3C-3H). The patient underwent quarantine for $15 \mathrm{~d}$ with daily

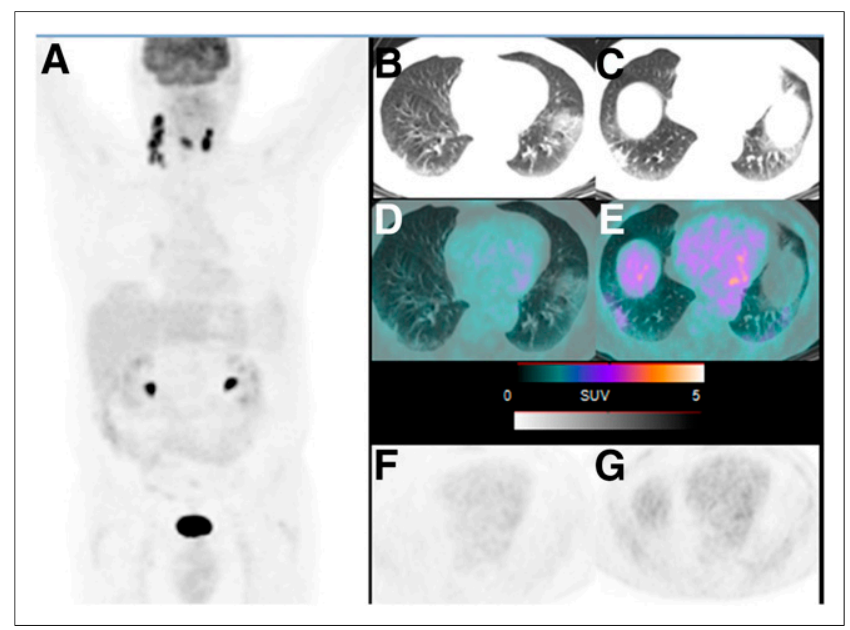

FIGURE 2. (A) Maximum-intensity projection showing increased ${ }^{18} \mathrm{~F}-$ FDG uptake at right epiglottis and cervical nodes. (B-G) Axial CT (B and $C), P E T / C T$ ( $D$ and $E$ ), and PET (F and $G$ ) images showing interstitial pneumonia with ${ }^{18} \mathrm{~F}-\mathrm{FDG}$-avid GGOs (SUV $\left.\mathrm{max}_{\max }, 2\right)$. 


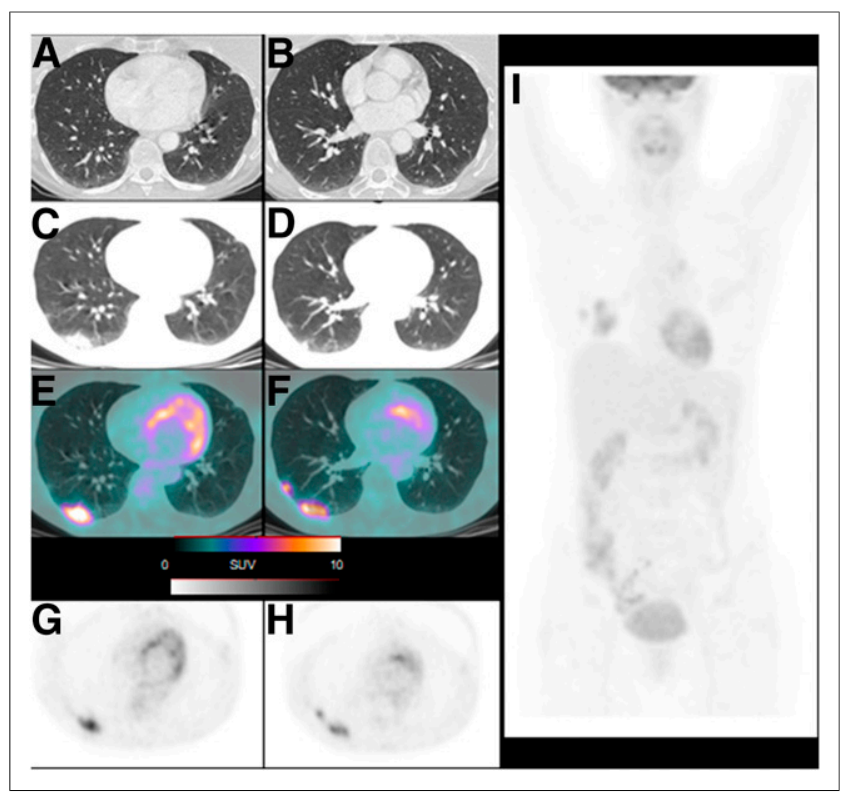

FIGURE 3. (A and B) High-resolution thoracic CT scan with no sign of pneumonia. (C-H) PET/CT images obtained 2 wk later, showing GGOs and consolidative areas in right inferior lobe on CT (C and D), PET/CT (E and $F$ ), and PET (G and $H$ ), with $S V_{\text {max }}$ of 7.7. (I) Maximum-intensity projection confirming pulmonary uptake.

body temperature measurements but remained asymptomatic during that time. She was subsequently scheduled for nodal biopsy.

\section{Case 4}

An asymptomatic 55-y-old woman with Hodgkin lymphoma treated previously with 2 lines of chemotherapy underwent a chest CT scan, which was positive for axillary nodes but without showing lung alterations (Figs. 4A and 4B). One week later, she underwent ${ }^{18} \mathrm{~F}$-FDG PET/CT, which confirmed active lymphoma in the axillary nodes and showed new interstitial opacities near the pleura of the right lung (Figs. 4C-4F). The patient underwent immediate RT-PCR testing, which was negative, but fever and dyspnea appeared several days later, leading to hospitalization and retesting, with proof of COVID-19.

\section{Case 5}

An asymptomatic 65-y-old man with a history of laryngeal cancer previously treated with surgery underwent follow-up PET/ CT, which was negative for malignancy. Thoracic CT displayed several GGOs showing increased radiotracer uptake $\left(\mathrm{SUV}_{\max }, 5.3\right)$ in the right lung (Figs. 5A-5F). Being asymptomatic, the patient did not undergo pharyngeal swabs but was strictly monitored by his general physician, who checked body temperature and clinical conditions for $15 \mathrm{~d}$ as suggested by national rules.

\section{Case 6}

An asymptomatic 65-y-old woman with a history of ovarian cancer previously treated with surgery plus chemotherapy underwent follow-up PET/CT, which was negative for ovarian malignancy but showed interstitial pneumonia with consolidative areas in both lungs and increased ${ }^{18} \mathrm{~F}$-FDG uptake (Figs. 6A-6F). Moreover, some mediastinal nodes were found to be ${ }^{18} \mathrm{~F}-\mathrm{FDG}-$ avid (Figs. 6G-6I). RT-PCR testing was performed immediately and confirmed COVID-19. Her husband also tested positive for COVID-19.

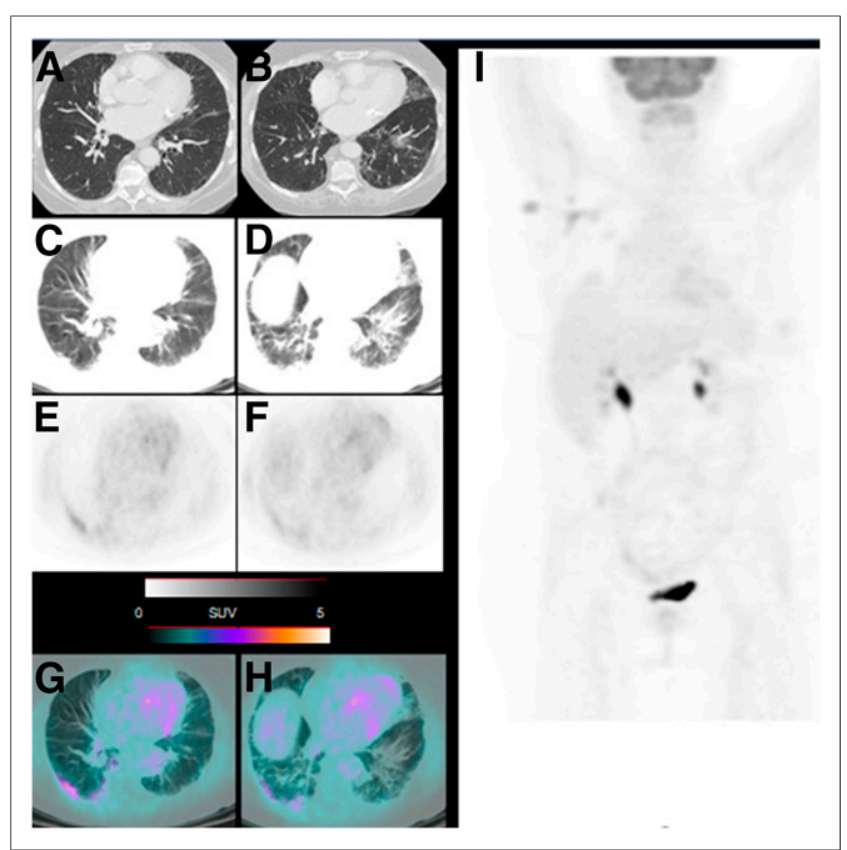

FIGURE 4. (A and B) High-resolution thoracic CT with no sign of pneumonia. (C-H) PET/CT performed 1 wk later, showing bilateral pneumonia with GGOs on CT (C and D), PET (E and F), and PET/CT ( $G$ and $H$ ) images, with $S U V_{\max }$ of 5. (I) Maximum-intensity projection showing ${ }^{18} \mathrm{~F}-\mathrm{FDG}-$ positive axillary nodal lesion.

\section{Case 7}

Among the 12 patients who received radioiodine for differentiated thyroid carcinoma, there was 1 patient in whom interstitial pneumonia was detected incidentally on ${ }^{131}$ I SPECT/CT. This 79y-old woman, with poorly differentiated thyroid carcinoma, had a prior chest CT scan showing bilateral solid lung nodules suggestive of metastases. She received $3.7 \mathrm{GBq}$ of ${ }^{131} \mathrm{I}$ for remnant ablation and assessment of the radioiodine positivity of the lung nodules. The serum thyroid-stimulating hormone level was $100 \mathrm{mU} / \mathrm{L}$, serum

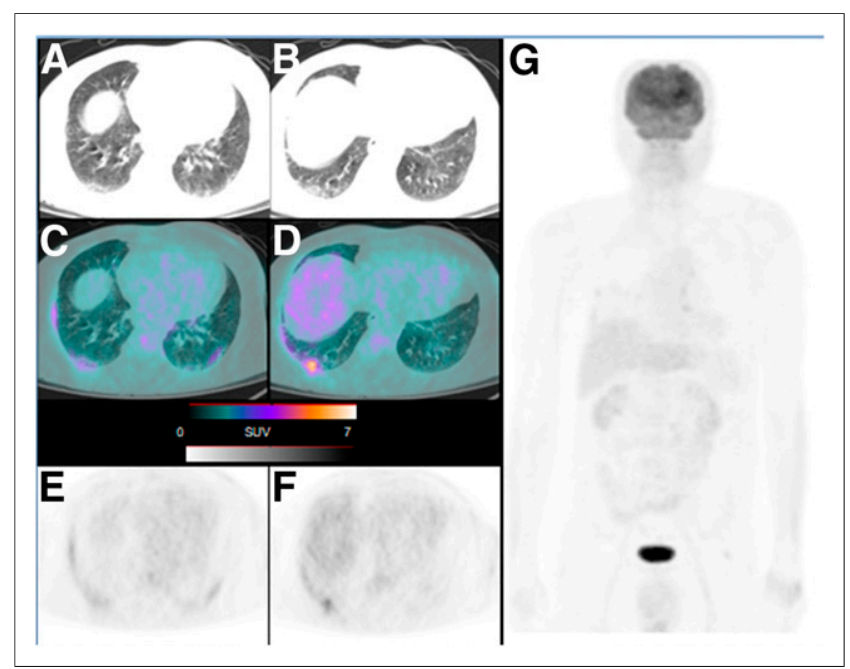

FIGURE 5. (A-F) Axial CT ( $A$ and $B$ ), PET/CT ( $C$ and $D$ ), and PET (E and F) images showing increased radiotracer uptake corresponding to interstitial lung alterations $\left(\mathrm{SUV}_{\max }, 5.3\right)$. (G) Maximum-intensity projection showing entire body. 


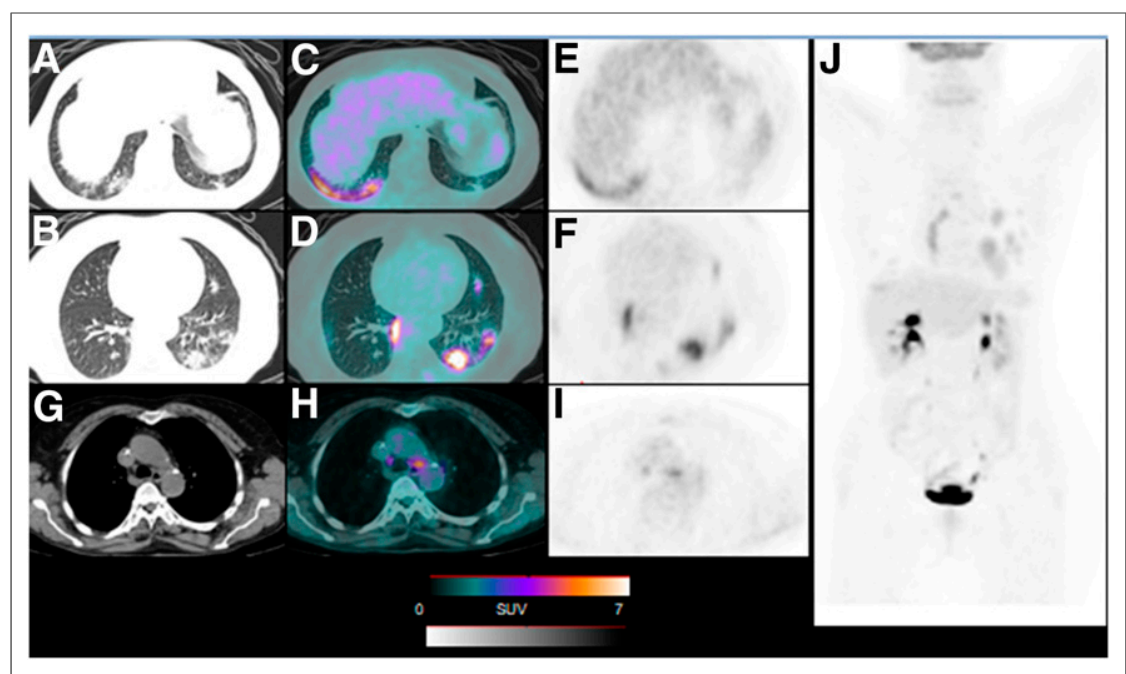

FIGURE 6. (A-I) Axial CT ( $A$ and B), PET/CT (C and D), and PET (E and F) images showing significant pneumonia with GGOs, especially in left lung (SUV $\left.\max _{\max }, 6.9\right)$, and ${ }^{18} \mathrm{~F}$-FDG-avid mediastinal nodes $(G-l)(J)$. Maximum-intensity projection confirming lung and nodal alterations. increase, occupying most of the lungs. The CT pattern is similar to that for other viral pneumonias, such as Middle East respiratory syndrome and SARS (13). The role of CT in screening for viral lung disease in asymptomatic subjects, however, is not well defined.

In our report, all lung lesions on CT were ${ }^{18} \mathrm{~F}-\mathrm{FDG}$-positive, and in 1 patient, ${ }^{18} \mathrm{~F}-\mathrm{FDG}$ avidity was identified even in the mediastinal nodes. This metabolically active pattern of COVID-19 pneumonia on ${ }^{18}$ F-FDG PET/ CT scans has been supported so far only by a cases series and a case report $(11,12)$. Qin et al. (12) described 4 cases of suspected COVID-19 pneumonia with increased ${ }^{18} \mathrm{~F}$ FDG uptake but no RT-PCR confirmation. In our population, 5 of 7 patients underwent RT-PCR, which confirmed COVID-19. In the other cases, RT-PCR was not performed because national rules did not require it in the absence of symptoms. Another difference from prior publications is that all our patients

thyroglobulin was higher than $2,500 \mathrm{ng} / \mathrm{mL}$, and serum thyroglobulin antibodies were $50.6 \mathrm{UI} / \mathrm{mL}$. After $72 \mathrm{~h}$, planar radioiodine scintigraphy (Figs. 7A and 7B) showed strong focal uptake in residual thyroid tissue but no other foci. SPECT/CT ruled out radioiodine avidity in the lung nodules, but the CT portion also showed new, diffuse interstitial pneumonia with peripheral GGOs, without increased radioiodine uptake (Figs. 7C and 7D). Fever and cough appeared $1 \mathrm{~d}$ after the scan. RT-PCR testing was positive for COVID-19, and probatory therapy with hydroxychloroquine plus ritonavir-lopinavir was started.

\section{DISCUSSION}

High-resolution chest $\mathrm{CT}$ is the routine, preferred tool for detection, diagnosis, and monitoring of symptomatic COVID-19 pneumonia. The CT findings for COVID-19 have been widely reported (2-6). Usually, single or multiple GGOs, nodules, or plaques may appear in the early phases. With progression of the disease, the lesions

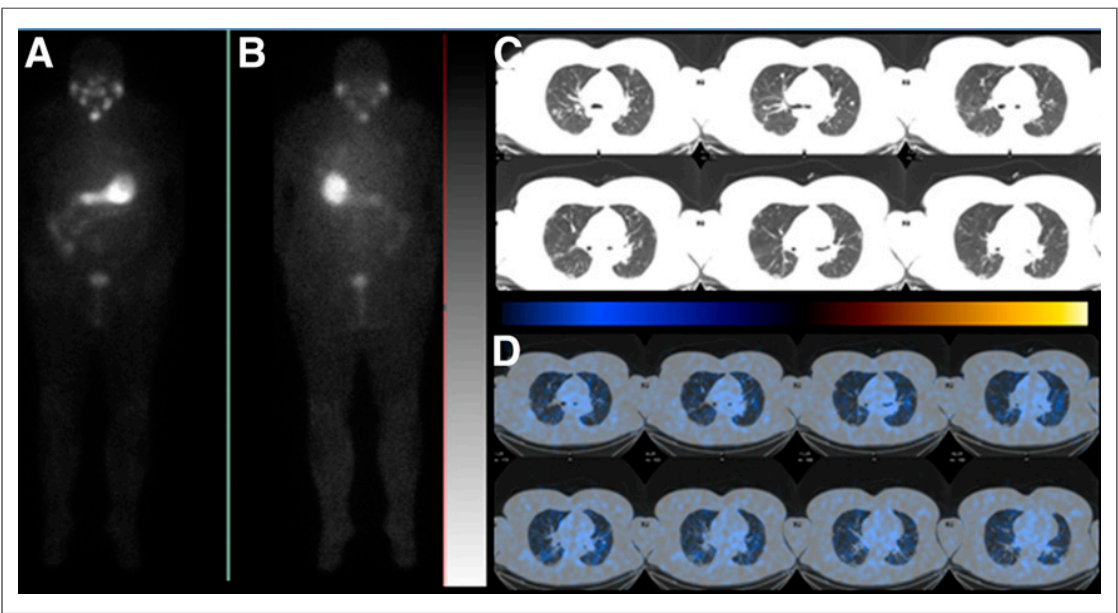

FIGURE 7. (A and B) Whole-body anterior (A) and posterior (B) scintigraphic images after radioiodine treatment showing focal uptake in neck consistent with thyroid remnant. (C and D) Several axial CT (C) and SPECT/CT (D) fused slices characterized by various bilateral GGOs without radioiodine uptake. were asymptomatic at the time of imaging and for $2 \mathrm{wk}$ beforehand (because only patients without signs or symptoms related to COVID19 for a 2-wk period were allowed to access the hospital). Thus, our case series confirms that it is possible to have COVID-19 pneumonia without symptoms, increasing the risk of spreading the infection.

Because the CT component of hybrid imaging sessions is used mainly for attenuation correction, its diagnostic quality is not entirely equivalent to that of dedicated chest CT. Specifically, a lack of full inspiration may contribute to higher density in the posterior lung fields. But metabolic activity may help to distinguish an infectious origin from an artifact, and a more widespread pattern of GGO across the lungs may also help to identify COVID-19 more specifically. It should be noted, however, that our report is observatory in nature and that a precise definition of the accuracy of PET findings of COVID19 would require prospective validation in larger cohorts.

Nevertheless, our observations show, on the one hand, that it is mandatory for health care personnel to use hygienic measures, minimize patient contact, optimize distance, and wear protective equipment for general clinical services in regions with a high COVID19 prevalence. In our department, health care personnel started to use individual protective equipment at the beginning of March, and no cases of infected staff have been reported to date. Moreover, our national laws do not require a swab test for asymptomatic staff who come into contact with COVID-19positive patients. On the other hand, during image interpretation, it is important to consider whether any findings might potentially be related to COVID-19 and to report them to the patient and the referring physician immediately. Depending on the current local regulations, an asymptomatic patient might be able to stay at home with adequate clinical monitoring, whereas if there is clinical doubt, more aggressive measures might be appropriate, with the risk of postponing oncologic treatments and potentially 
affecting survival. Thereby, the incidental detection of COVID-19 pneumonia in early stages may help prevent further spread of the virus.

\section{CONCLUSION}

COVID-19-associated pneumonia is ${ }^{18}$ F-FDG-avid and may be detected as an incidental finding in asymptomatic patients undergoing nuclear medicine examinations for oncologic indications in regions with a high COVID-19 prevalence. Nuclear medicine services should be aware of this possibility and prepare accordingly.

\section{DISCLOSURE}

No potential conflict of interest relevant to this article was reported.

\section{KEY POINTS}

QUESTION: What is the possible impact of nuclear medicine examinations in the management of COVID-19 patients?

PERTINENT FINDINGS: A significant number of incidental pneumonia suspected of stemming from COVID-19 was discovered in patients undergoing PET/CT and SPECT/CT scans.

IMPLICATIONS FOR PATIENT CARE: Nuclear medicine examinations may help to detect incidentally COVID-19-associated pneumonia in asymptomatic oncologic patients.

\section{REFERENCES}

1. Park M, Thwaites RS, Openshaw PJM. COVID-19: lessons from SARS and MERS. Eur J Immunol. 2020;50:308-311.

2. Huang C, Wang Y, Li X, et al. Clinical features of patients infected with 2019 novel coronavirus in Wuhan, China. Lancet. 2020;395:497-506.

3. Kanne JP, Little BP, Chung JH, Elicker BM, Ketai LH. Essentials for radiologists on COVID-19: an update-Radiology scientific expert panel. Radiology. February 20, 2020 [Epub ahead of print].

4. Shi H, Han X, Jiang N, et al. Radiological findings from 81 patients with COVID-19 pneumonia in Wuhan, China: a descriptive study. Lancet Infect Dis. 2020;20:425-434.

5. Lee EYP, Ng MY, Khong PL. COVID-19 pneumonia: what has CT taught us. Lancet Infect Dis. 2020;20:P384-P385.

6. Chung M, Bernheim A, Mei X, et al. CT imaging features of 2019 novel coronavirus (2019-nCoV). Radiology. 2020;295:202-207.

7. Lan L, Xu D, YeG, et al. Positive RT-PCR test results in patients recovered fromCOVID-19. JAMA. February 27, 2020 [Epub ahead of print].

8. Fang Y, Zhang H, Xie J, et al. Sensitivity of chest CT for COVID-19: comparison to RT-PCR. Radiology. February 19, 2020 [Epub ahead of print].

9. Xie X, Zhong Z, Zhao W, Zheng C, Wang F, Liu J. Chest CT for typical 2019nCoV pneumonia: relationship to negative RT-PCR testing. Radiology. February 12, 2020 [Epub ahead of print].

10. Deng Y, Lei L, Chen Y, Zhang W. The potential added value of FDG PET/CT for COVID-19 pneumonia. Eur J Nucl Med Mol Imaging. March 21, 2020 [Epub ahead of print].

11. Zou S, Zhu X. FDG PET/CT of COVID-19. Radiology. March 6, 2020 [Epub ahead of print].

12. Qin C, Liu F, Yen TC, Lan X. ${ }^{18}$ F-FDG PET/CT findings of COVID-19: a series of four highly suspected cases. Eur J Nucl Med Mol Imaging. 2020;47:1281-1286.

13. Koo HJ, Lim S, Choe J, et al. Radiographic and CT features of viral pneumonia. Radiographics. 2018;38:719-739. 